\title{
TERRITOIRE ET ENVIRONNEMENT Maîtrise des flux de polluants sur les territoires agricoles
}

\section{Farmland and the environment Controlling the flow of pollutants on agricultural land}

Oléagineux, Corps Gras, Lipides. Volume 7, Numéro 6, 512-5, Novembre - Décembre 2000, Dossier : "Agriculture, recherche et territoire"

Auteur(s) : Philippe Martin, Ina-PG, UMR SADAPT, 16, rue Claude-Bernard, 75231 Paris Cedex 05, France.

Résumé : La maîtrise des flux de polluants demande une identification correcte de l'espace physique à l'origine des problèmes ainsi que des territoires d'exploitation agricole à l'intérieur de cet espace physique. L'exemple de la pollution diffuse par les nitrates montre qu'il existe de multiples méthodes de diagnostic et que le choix d'une méthode dépend avant tout de l'objectif poursuivi. Pour être acceptées, les propositions de modifications doivent prendre en compte les contraintes des agriculteurs mais aussi celles des services de développement agricole qui auront à les diffuser. La prise de conscience des problèmes par l'agriculteur ainsi que la confiance entre agriculteurs et services de développement sont des facteurs déterminants en ce domaine. De son côté, la recherche se doit de produire de nouvelles méthodes de diagnostic permettant notamment de hiérarchiser les risques environnementaux au sein d'un territoire d'exploitation inclus dans un espace physique donné.

Mots-clés : pollution, changement de pratiques, territoires, agriculture.

Summary : The control of pollutant flows requires correct identification of environmental factors where the pollution originates and the agricultural territories within that physical context. Even if many diagnostic methods are now available for non point source nitrate pollution characterisation, the choice of a specific method depends on the objectives pursued. To be accepted, proposed changes in practice must take into account the constraints of both the farmers and the extension services in charge of the problem. Farmer recognition of the importance of agricultural pollution and level of trust between farmers and extension workers are determining factors. Research must produce new diagnostic methods making it possible to treat environmental risks within an agricultural territory on a hierarchical basis.

Keywords : pollution changes in practices, territories, agriculture 


\section{ARTICLE}

\section{Contexte}

\section{De l'espace physique aux territoires d'acteurs}

Les problèmes environnementaux liés à l'agriculture, bien que très diversifiés (pollution des eaux, dégradation paysagère et écologique, etc.), ont souvent pour point commun une forte composante spatiale. On peut ainsi identifier des lieux d'injection de substances indésirables dans le milieu ou de prélèvement de ressources qui vont avoir des répercussions néfastes dans d'autres lieux. C'est, par exemple, un ruissellement de surface sur des parcelles qui va générer des inondations dans une agglomération en aval ou encore de l'eau pompée dans une nappe qui va entraîner, quelques kilomètres plus loin, l'assèchement d'un cours d'eau.

Dans le cas de polluants entraînés par l'eau, l'identification correcte de l'espace physique à prendre en compte pour traiter des problèmes environnementaux est une étape essentielle mais qui n'est pas toujours facile à réaliser. S'il est relativement aisé de définir un bassin versant d'alimentation d'un cours d'eau de surface, il est souvent plus difficile de bien délimiter un bassin d'alimentation d'une nappe profonde. Une fois l'espace physique identifié, il faut repérer les différents découpages territoriaux. Par territoire on entend des portions d'espace sur lesquelles un acteur individuel ou collectif exerce une activité. En amont des problèmes, on a les territoires d'exploitation qui correspondent aux ensembles de parcelles exploitées par un même agriculteur. En aval, on a des territoires publics (voirie communale, départementale) ou privés (habitations) qui subissent souvent l'essentiel des nuisances et des dégâts. Les différents territoires sont bien souvent gérés indépendamment les uns des autres hormis quelques règles de bon voisinage imposées par les pouvoirs publics (plan d'occupation des sols, périmètre de protection de captage, etc.). La plupart du temps, il n'existe pas de gestionnaire global de l'espace concerné ; l'action concertée n'est donc pas chose courante alors qu'elle est souvent nécessaire à la résolution des problèmes.

\section{Activités agricoles et environnement : l'agriculteur n'est pas le seul décideur}

Les activités agricoles peuvent être classées en deux grandes catégories. La première correspond à des modifications du milieu relativement peu fréquentes et difficilement réversibles qui concernent la taille, la forme, la nature des limites de parcelles ainsi que les maillages hydrologiques (remembrement, réalisation de fossés, bandes enherbées, haies, etc.). La deuxième est constituée de pratiques, plus fréquentes mais à effets moins marqués, de conduite des champs et des troupeaux sans oublier les milieux interstitiels que sont les bordures de champ et les fossés [1]. Les décisions portant sur les conduites des champs et des troupeaux relèvent directement des agriculteurs. Les décisions qui portent sur les structures paysagères, si elles restent pour partie prises par des agriculteurs, le sont aussi par les propriétaires, les communes, les administrations, ce qui complique d'autant leur mise en œuvre.

\section{Approche agronomique des relations entre territoires d'exploitation et flux de polluants}

L'analyse des relations entre territoires d'exploitation et flux de polluants passe par une démarche pluridisciplinaire depuis l'hydrogéologue, qui définit la circulation des eaux dans un espace physique, jusqu'au sociologue, qui s'intéresse aux relations entre les agriculteurs et les autres acteurs. Dans les lignes qui suivent nous développerons plus particulièrement la contribution de la discipline 
agronomique à cette analyse en nous cantonnant à la deuxième catégorie d'activités agricoles (pratiques culturales) qui relèvent le plus directement de la responsabilité des agriculteurs.

\section{Deux entrées possibles : l'espace physique et le territoire d'exploitation}

On peut aborder les relations entre territoires d'exploitation et flux de polluants par l'espace physique ou par le territoire d'exploitation. L'entrée par l'espace physique consiste à identifier l'impact de chaque territoire ou portion de territoire d'exploitation présent sur cet espace. Cette approche s'appuie sur le caractère fortement spatialisé des phénomènes et sur la nécessité d'intégrer les relations entre les différents territoires pour résoudre les problèmes. L'entrée par les territoires d'exploitation consiste à analyser, sur un territoire d'exploitation donné, différentes questions environnementales quasi indépendamment de ce qui se passe sur les autres territoires. Cette deuxième approche est typiquement ce qui va se développer dans le cadre des CTE ou d'une qualification des exploitations agricoles de type " agriculture raisonnée ", alors que la première est plus proche d'une démarche de type FERTI-Mieux développée par la profession agricole.

Quelle que soit l'entrée retenue, par l'espace physique ou par le territoire d'exploitation, l'analyse agronomique reste la même. Elle vise tout d'abord à faire un diagnostic en identifiant les systèmes de culture à risques pour la ou les thématiques environnementales abordées, puis à voir la part de ces systèmes de culture à risque dans la ou les exploitations étudiées. Dans un deuxième temps, on doit formuler des propositions acceptables de modifications individuelles et/ou collectives pour améliorer la situation.

\section{La démarche au travers d'un exemple}

Partant de la méthode générale présentée ci-dessus nous illustrerons chacune des étapes pour le cas de la pollution diffuse par les nitrates.

\section{Analyse des systèmes de culture à risque}

Le principe de base est d'identifier les éléments des systèmes de culture qui déclenchent ou amplifient les mécanismes à l'origine des flux de polluants. Ceci nécessite de définir clairement le système étudié dans ses dimensions spatiales et temporelles. La pollution diffuse par les nitrates commence lorsque les précipitations entraînent les nitrates de la solution du sol en dessous de la profondeur maximale d'enracinement. La dimension temporelle du système est définie, d'une part, par les cycles de précipitation (à l'origine du lessivage) et de température (qui détermine l'intensité de la minéralisation) et, d'autre part, par les cycles de végétation (qui font varier les prélèvements instantanés en azote ainsi que la profondeur d'enracinement de ces peuplements) et d'apport en éléments azotés (qui font varier la quantité d'azote dans le milieu). La dimension spatiale n'a longtemps été prise en compte qu'au travers des différences de types de sol (à l'origine d'un lessivage différentiel et d'une variabilité des rendements plus ou moins forte). Actuellement, l'intégration de connaissances sur la circulation de l'eau sous la profondeur maximale d'enracinement conduit aussi à considérer différemment des parcelles à l'aplomb de failles géologiques, permettant une circulation directe avec la ressource en eau à protéger, et d'autres parcelles, situées sur des zones non faillées en amont de mares où s'opère une dénitrification. 


\section{Part de ces systèmes de culture à risque dans la ou les exploitations étudiées}

Différentes approches ont été proposées pour caractériser le caractère polluant d'une exploitation ou d'un ensemble d'exploitations agricoles. Certaines approches très globales ne prennent que très peu en compte les éléments de diagnostic sur les systèmes de culture présentés ci-dessus. La méthode du bilan à l'exploitation [2] fait partie de celles-ci puisqu'elle caractérise le caractère polluant d'une exploitation par la simple différence entre les entrées et les sorties d'azote au niveau de l'exploitation. Les entrées sont constituées par les achats d'engrais, d'aliment pour le bétail ainsi que par les importations de fumier et lisier en provenance d'autres exploitations. Les sorties correspondent aux exportations de produits végétaux et animaux en dehors de l'exploitation. D'un point de vue agronomique, cette méthode fait l'impasse sur de nombreux phénomènes. Un bilan en apparence équilibré peut masquer des problèmes liés à la coexistence de parcelles excédentaires et déficitaires en azote au sein d'une même exploitation. D'un autre côté, ne sont pas prises en compte les pertes d'azote telles que celles liées à la volatilisation d'une partie des effluents sur leur lieu de stockage. En fait, l'intérêt de cette méthode réside avant tout dans sa facilité de mise en œuvre et de contrôle (fondée avant tout sur des documents comptables) qui fait que l'administration s'y intéresse de près dans la perspective d'une éventuelle « redevance azote ".

Le bilan CORPEN [3] est aussi une méthode de bilan global réalisée non au niveau de l'exploitation mais au niveau des surfaces exploitées. On évite ainsi, pour la pollution diffuse, les erreurs de bilan liées à des problèmes du type "volatilisation des effluents avant épandage ". En revanche, les problèmes liés à la coexistence de parcelles déficitaires avec des parcelles excédentaires demeure. Une autre difficulté tient au fait que le bilan CORPEN, tout comme le bilan à l'exploitation, est calculé pour une année donnée et, de ce fait, ne prend pas en compte les phénomènes de minéralisation s'effectuant sur plusieurs années comme ceux liés à des retournements de prairies. C'est pour mieux intégrer cette dynamique temporelle que Benoît [4] a développé l'indicateur BASCULE permettant de prendre en compte les successions de culture mises en œuvre sur les parcelles.

Il existe encore d'autres méthodes [5] que nous ne présenterons pas et qui permettent d'établir le caractère plus ou moins polluant de tout ou partie d'un territoire d'exploitation. L'intérêt de l'une ou l'autre de ces méthodes dépend avant tout de l'objectif poursuivi. Quand l'objectif est plutôt de caractériser le risque environnemental pour une exploitation donnée, on pourra se permettre d'aller dans le détail des pratiques, voire des raisonnements associés à ces pratiques [6], alors que, lorsque l'objectif est la caractérisation du caractère polluant d'un grand parc d'exploitations agricoles, on s'orientera vers des méthodes nécessitant moins d'informations à la base (de type bilan à l'exploitation) mais qui permettent néanmoins de mettre en évidence les situations les plus critiques sur lesquelles devra se concentrer l'action dans un premier temps.

\section{Formulation de propositions de modifications individuelles et/ou collectives}

Pour être acceptables les modifications proposées doivent intégrer les contraintes propres aux exploitations agricoles sans toutefois se laisser enfermer dans la logique actuelle de fonctionnement des exploitations. De fait, deux écueils sont à éviter : concevoir l'innovation comme un processus linéaire (les chercheurs conçoivent des solutions techniques, les agriculteurs les mettent en œuvre) et, au contraire, admettre, sans réserve, que les agriculteurs ont de bonnes raisons de faire ce qu'ils font... Dans les deux cas on arrive à un blocage : soit que la solution technique proposée n'intègre pas une contrainte majeure liée, par exemple, à l'organisation du travail sur l'exploitation ou au 
mode de raisonnement du conseiller agricole, soit que le fonctionnement actuel de l'exploitation repose sur une erreur technique que l'agriculteur serait prêt à reconnaître pour peu qu'on la lui explique. Partant de là, il serait logique que les propositions de modifications de pratiques faites aux agriculteurs intègrent les objectifs spécifiques que ces agriculteurs assignent eux-mêmes à ces pratiques. Un travail récent [7] va dans ce sens en montrant que les agriculteurs ont des objectifs variables pour la conduite de leurs intercultures (maîtrise des adventices, épandage d'effluents, correction de dégradation de l'état structural, etc.) et qu'il faut donc proposer des solutions adaptées à chacun de ces objectifs pour lutter contre la pollution diffuse par les nitrates.

\section{Les facteurs psycho-sociologiques du changement des pratiques}

L'existence de solutions techniques valables pour la protection de l'environnement n'assure pas leur adoption par les intéressés, encore faut-il qu'elles soient reprises par les structures de conseil privées et publiques et que les agriculteurs les adoptent.

\section{Vers une responsabilisation des agriculteurs}

La prise de conscience du problème par les agriculteurs est une étape nécessaire à la modification des pratiques. Cette prise de conscience est souvent freinée par une minimisation des problèmes environnementaux de la part des agriculteurs. C'est ainsi que Cattan et Mermet [8] notent, à partir d'enquêtes réalisées au début des années 90 , que chaque agriculteur a tendance à minimiser davantage le problème qui le concerne le plus. Pour les exploitants en grande culture, qui les utilisent avec parcimonie, les pesticides sont de loin les intrants les plus dangereux. Pour les arboriculteurs, gros utilisateurs, les pesticides ne sont pas si dangereux (ils prennent d'ailleurs moins de précautions d'utilisation). La reconnaissance de la responsabilité tient aussi à la localisation du territoire d'exploitation dans l'espace physique [9]. Les études sur le ruissellement érosif ont ainsi pu montrer des différences entre les agriculteurs situés à l'aval des bassins versants, là où le ruissellement se concentre et érode les terres, et ceux dont les terres sont situées en amont, là où se crée le ruissellement qui ne générera des problèmes qu'en aval [10]. Ceux de l'aval sont fortement sensibilisés aux techniques de lutte anti-érosives alors que l'amont, où une modification des pratiques serait la plus nécessaire, n'en voit pas l'intérêt. II peut en être de même pour les problèmes de pollution diffuse par les nitrates des nappes profondes [9]. Quand la maison d'habitation ou les bâtiments d'élevage de l'agriculteur sont alimentés avec de l'eau très chargée en nitrates, les agriculteurs sont plus enclins à se sentir responsables que lorsqu'ils ne sont pas alimentés par cette eau. Cette dernière situation est d'autant plus dangereuse que la nappe qui alimente l'habitation n'est pas celle sur laquelle se trouve le territoire d'exploitation et que cette dernière nappe est très chargée en nitrates.

\section{Des structures de conseil en mutation}

La prise de conscience des agriculteurs peut aussi être freinée par l'existence de messages divergents entre les structures de développement et les structures de négoce. On peut citer l'exemple des doses d'intrants préconisées. Elles sont souvent plus élevées du côté du négoce que des chambres d'agriculture. Ceci amène les agriculteurs à « faire la part des choses » en adoptant parfois des doses intermédiaires. D'un côté, l'agriculteur sait que le négoce cherche avant tout à vendre son produit et, d'un autre, il ne fait pas entièrement confiance aux conseillers des chambres d'agriculture qui sont soupçonnés (à tort ou à raison) de mettre plus en avant la protection de l'environnement que la 
protection du revenu de l'agriculteur. Cattan et Mermet [8] notent que les conseillers des chambres, conscients du malaise, cherchent à limiter les risques de se discréditer auprès des agriculteurs dont ils perdraient la confiance. Pour cela, ils limitent de fait les pratiques conseillées à celles n'entraînant pas de réduction potentielle du revenu des agriculteurs. Ceci fait que, dans un contexte de grande diversité de situations de terrains et d'activités agricoles, alors qu'on devrait s'attendre à une diversité de propositions en direction des agriculteurs qui, seuls pilotes à bord, seraient en mesure de choisir, seules quelques mesures sont retenues par les organismes de développement. L'amélioration de la situation environnementale en est d'autant ralentie que ces mesures ne sont pas toujours les plus efficaces, mais les structures de conseil gardent la confiance des agriculteurs.

\section{Quelques pistes de réflexion pour l'avenir}

\section{Du « pollueur » au « pollué »}

Dans tout ce qui précède, nous avons considéré que l'agriculteur était l'émetteur de polluant dont le reste de la société subit les effets. II peut aussi arriver que les rôles soient inversés, c'est-à-dire que les territoires agricoles soient les récepteurs de pollutions (réelles ou potentielles) et de nuisances émises par le reste de la société. La valorisation agronomique des déchets urbains et industriels relève de ce domaine, de même que l'utilisation du territoire agricole amont comme champ d'expansion des crues d'un cours d'eau pour protéger une agglomération située plus en aval [11]. Notons que l'approche agronomique serait très proche de ce qui a été présenté ci-dessus. Il faudrait en effet, dans un premier temps, classer les systèmes de culture par rapport à leur sensibilité à l'agent extérieur (polluant, submersion plus ou moins longue) avant de proposer des modifications. Notons que, dans ce cas, les modifications porteront plus facilement sur les doses et fréquences d'apports extérieurs que sur les pratiques de l'agriculteur ou que, tout au moins si changement nécessaire de pratique il y a suite aux apports extérieurs, une compensation financière sera à envisager.

\section{Vers une amélioration des outils et méthodes}

La maîtrise des flux de polluants sur les territoires agricoles fait intervenir de nombreux acteurs. Concernant les agriculteurs, il devient nécessaire de disposer d'outils permettant de porter des diagnostics sur les pratiques culturales à différentes échelles (parcelle, exploitation, bassin versant, etc.). De fait, il faudrait développer pour les différents types de pollution et de nuisance (phytosanitaires, coulées boueuses, etc.) des batteries d'indicateurs du type de ce que nous avons présenté pour l'azote. Une même pratique culturale agissant (pas toujours dans le même sens) sur plusieurs sources de polluant, il faudra aussi développer des outils permettant de hiérarchiser les risques sur un territoire d'exploitation afin d'aider l'agriculteur à prendre ses décisions. Enfin, la maîtrise effective des flux de polluants nécessitera vraisemblablement une coopération accrue entre chercheurs et agents de développement, d'une part, et entre disciplines techniques et sciences sociales, d'autre part. 


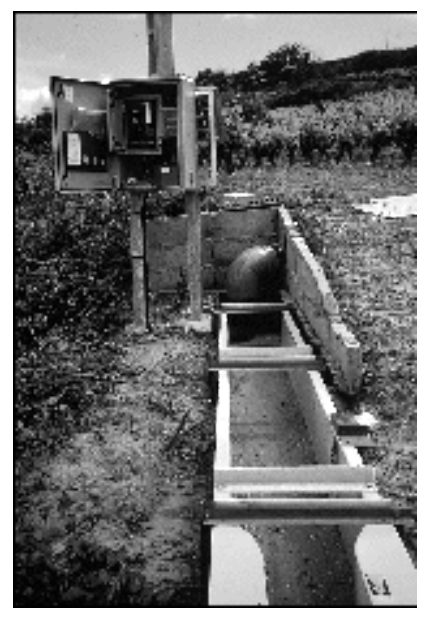

Dispositif de mesure du ruissellement à l'échelle planétaire (photo P. Andrieux, Inra)

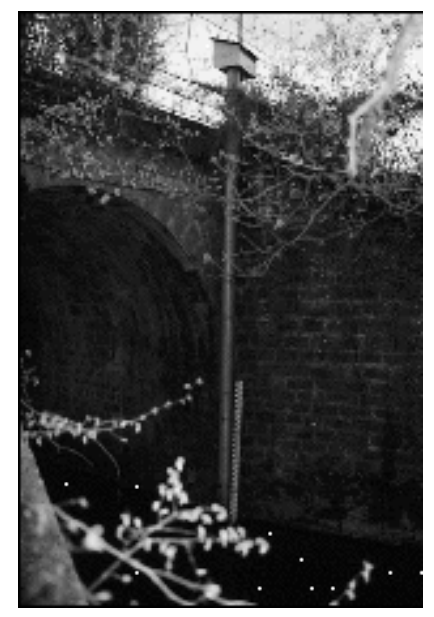

Bassin versant du Foron.

Dispositif de mesure

(photo C. Slagmulder, Inra)

\section{REFERENCES}

1. THENAIL C, LE CCEUR D, BAUDRY J (2000). Relationships between field boundaries, farming systems and landscape : consequences on biodiversity pattern in agrarian landscapes. 4th European symposium on European farming and rural systems research and extension : environmental, agricultural and socio-economic issues. Volos, Association for Farming System Research and Extension, European group : 63-76.

2. SIMON JC, LE CORRE L (1992). Le bilan apparent de l'azote à l'échelle de l'exploitation : méthodologie, exemples de résultats. Fourrage, $129: 79-94$.

3. CORPEN (1988). Bilan de l'azote à l'exploitation. Programme d'action en Bretagne.

4. BENOîT M (1992). Un indicateur des risques de pollution nommé BASCULE (Balance Azotée Spatialisée des systèmes de CULture de l'Exploitation). Courrier de la cellule environnement de I'Inra, 18.

5. BOCKSTALLER C, GIRARDIN P (1998). Mode de calcul d'un indicateur agro-écologique : I'indicateur " azote ». In : Association pour la relance agronomique en Alsace, Inra, Colmar.

6. AUBRY C, BIARNES A, MAXIME F, PAPY F (1998). Modélisation de l'organisation technique de la production dans l'exploitation agricole : la constitution de systèmes de culture. Etud Rech Syst Agraires Dév, 31 : 25-43.

7. RABAUD A (2000). Analyse des modes de gestion de l'interculture en Pays de Loire. Mémoire de DAA de I'Ina-PG, dpt AGER.

8. CATTAN A, MERMET L (1998). Dix ans de promotion des pratiques agricoles favorables à l'environnement. In : BARRAQUÉ B, THEYS J, éd. Les politiques d'environnement. Évaluation de la première génération : 1971-1995. Recherches : 307-30. 
9. BENOÎT M, PAPY F (1997). Pratiques agricoles et qualité de l'eau sur le territoire alimentant un captage. In : L'eau dans l'espace rural, production végétale et qualité de l'eau. Collection "Mieux comprendre ", Universités francophones, Inra éd. : 323-36.

10. PAPY F, POUJADE C, SOUCHERE V (1991). Maîtrise du ruissellement et de l'érosion sur un territoire agricole (le double découpage de l'espace). Inra-SAD, Paris Grignon. Gestion de l'espace rural et systèmes d'information géographique. Séminaire INRA Florac, 22-24, octobre 1991 : 167-76.

11. PIVOT JM, JOSIEN E, TESTUT M, MARTIN P, GENDREAU N (2000). Flood hazard change and farmland vulnerability. European Conference on advances in flood research. Novembre 1-3, 2000Postdam (in press). 